\title{
Aggregate information, common knowledge and agreeing not to bet
}

\author{
Elias Tsakas
}

Accepted: 18 February 2010 / Published online: 7 March 2010

(C) The Author(s) 2010. This article is published with open access at Springerlink.com

\begin{abstract}
This note considers gambles that take place even if only some-but not all-individuals agree to participate. I show that the bet cannot take place if it is commonly known how many individuals are willing to participate.
\end{abstract}

Keywords Gambles · Limited participation · Common knowledge

\section{Introduction}

Aumann (1976) was the first one to formalize the concept of common knowledge. In his seminal paper he showed that if two people with a common prior have commonly known posterior probability assessments about an event, these probabilities are identical. Geanakoplos and Polemarchakis (1982) introduced the problem into a dynamic framework, by showing that if two individuals communicate their probability assessments back and forth and update accordingly, they will eventually agree on a common posterior probability.

Sebenius and Geanakoplos (1983) extended Aumann's result to expectations, by proving that if two people's expectations about a random variable are commonly known, then they are necessarily equal. A number of generalizations appeared in the literature ever since (Nielsen et al. 1990; Nielsen 1995; Hanson 1998, 2002). A direct application of this proposition is the famous no-bet theorem, which states that two riskaverse individuals with a common prior will never agree to participate in a gamble, if

\footnotetext{
E. Tsakas $(\bowtie)$

Department of Economics, Maastricht University, P.O. Box 616, 6200 MD Maastricht,

The Netherlands

e-mail: e.tsakas@maastrichtuniversity.nl

E. Tsakas

Department of Economics, Göteborg University, P.O. Box 640, 40530 Göteborg, Sweden
} 
their willingness to bet is commonly known. Milgrom and Stokey (1980) had already addressed this problem, by showing that common knowledge precludes trading among risk-averse agents in an uncertain environment.

This note extends the no-bet theorem to cases where the gamble can take place even if not everybody is willing to participate. Consider for instance a soccer game between $\mathrm{A}$ and $\mathrm{B}$, and suppose that Ann predicts that A will win, Bob predicts that B will win, while Carol predicts a draw. In order to participate in the bet, they have to pay a onedollar entry fee and the one who predicts correctly takes it all. If however Carol refuses to participate in the gamble, while Ann and Bob accept, the bet can still take place, with the prize being equal to 2 , instead of 3 dollars now. The only difference is that if a draw occurs nobody will win the prize and they will receive their entry fees back.

There is an important feature in this type of bets. The willingness to participate depends, not only on the private information, but also on who you are playing against. In the previous example, suppose that Ann believes that the probability of a draw is higher than A's victory. Then, if Carol stays in the bet, Bob does not, and Ann knows these two facts, then she rejects participation. The reason why she accepts to gamble against Bob, but not against Carol is that she believes that the probability of B winning is lower than the probability of A winning, which is lower than the probability of a draw. That is, her decision about participating in the gamble with exactly one opponent depends on who this opponent is, i.e., it depends on the identity of the other participant.

What is not straightforward from the previous analysis is the answer to the following question: What would Ann do if she knew that one more person was willing to gamble but she did not know who? In this case, she would form beliefs-given her private information - about who the other player was and she would maximize her expected payoff in a Bayesian manner. However, if she knew that everybody knew how many people were willing to participate, her beliefs about who the other player was would depend on what she believed about the other two people's beliefs, and so on.

In this note I show that the bet will not take place if the number of people who are willing to participate is commonly known. This result is quite surprising, since the expected payoff, and therefore the decision about whether to participate or not depends on the identity of the other participants. However, for the bet not to take place it is sufficient to have common knowledge of the aggregate behavior (how many people participate), instead of the individual behavior (who are the other participants).

This result can be easily extended to negative-sum bets, e.g., in a lottery where the prize depends on the number of participants, but not on who participates. In this case, if nobody has bought the winning coupon, the participants do not receive their entry fee back. Then, it is trivial to extend our main result and show that common knowledge of the number of participants precludes betting.

\section{Knowing how many players participate and agreeing not to bet}

\subsection{Information and knowledge}

Consider a finite state space $\Omega$, and a population $N=\{1, \ldots, n\}$. The measure $\pi$ determines the (common) prior beliefs of the individuals in the population over $\Omega$ 
and is assumed to assign positive probability to every state: $\pi(\omega)>0$ for every $\omega \in \Omega$. Every individual is endowed with an information partition $\mathcal{P}_{i}$ over $\Omega$. The set $P_{i}(\omega) \in \mathcal{P}_{i}$ denotes the element of $\mathcal{P}_{i}$ that contains $\omega$, and contains the states that $i$ cannot distinguish from $\omega$, with $\omega$ itself being one of those. Let $\mathcal{M}=\wedge_{i=1}^{n} \mathcal{P}_{i}$ denote the meet (finest common coarsening) of the information partitions, with $M(\omega)$ denoting the element of $\mathcal{M}$ that contains $\omega$. We define knowledge as usual, i.e., we say that $i$ knows some $E \subseteq \Omega$ at $\omega$ whenever $P_{i}(\omega) \subseteq E$. The event $E$ is commonly known at $\omega$ whenever $M(\omega) \subseteq E$. Throughout the paper we denote the actual state of the world by $\omega^{*}$. When we say that $E$ is common knowledge, we mean that it is commonly known at $\omega^{*}$.

\subsection{Gambles with limited participation}

We define a gamble that allows for limited participation as a collection $\mathcal{G}:=$ $\left\{G_{1}, \ldots, G_{n}\right\}$ of (possibly empty) disjoint subsets which cover $\Omega$, where $G_{i}$ denotes the set of states where $i$ wins. Participating in the gamble has a fixed cost (entry fee), which for simplicity and without loss of generality is normalized to 1 unit. Let $A_{i}=\{0,1\}$ denote $i$ 's action space: $i$ plays 1 when she is willing to participate in the bet and 0 when she is not. Let the action function $a_{i}: \Omega \rightarrow A_{i}$ determine the action that player $i$ undertakes at every state $\omega$. A natural assumption is that $a_{i}$ is $\mathcal{P}_{i}$-measurable, i.e., $a_{i}\left(\omega^{\prime}\right)=a_{i}(\omega)$ for all $\omega^{\prime} \in P_{i}(\omega)$, implying that $i$ knows what she is doing at all states. Let

$$
S(\omega):=\left\{i \in N: a_{i}(\omega)=1\right\}
$$

denote the set of people who agree to participate at $\omega$, and $s(\omega)$ be the cardinality of $S(\omega)$. The set

$$
B_{i}:=\{\omega \in \Omega: i \in S(\omega)\}=\left\{\omega \in \Omega: a_{i}(\omega)=1\right\}
$$

contains the states where $i$ participates in the bet.

Each agent's monetary payoff at some state $\omega$ depends on:

- the structure of the bet, i.e., who wins at $\omega$, and

- the action function of all agents, i.e., who participates at $\omega$.

If $i$ does not participate in the bet ( $i \notin S(\omega)$ ), her payoff is equal to 0 . If $i$ on the other hand participates in the bet $(i \in S(\omega))$, her payoff is equal to $s(\omega)-1$ if she wins, -1 if another participant wins and 0 if the winner has chosen not to participate. Formally, if $i$ 's monetary payoff at $\omega$ is equal to

$$
\begin{aligned}
V_{i}(\omega) & :=\mathbb{1}_{B_{i}}(\omega)\left(s(\omega) \mathbb{1}_{G_{i}}(\omega)-\mathbb{1}_{\cup_{j \in S(\omega)} G_{j}}(\omega)\right) \\
& =\mathbb{1}_{B_{i}}(\omega)\left(s(\omega) \pi\left(G_{i} \mid \omega\right)-\pi\left(\bigcup_{j \in S(\omega)} G_{j} \mid \omega\right)\right)
\end{aligned}
$$




$$
=\mathbb{1}_{B_{i}}(\omega)\left(s(\omega) \pi\left(G_{i} \mid \omega\right)-\sum_{j \in S(\omega)} \pi\left(G_{j} \mid \omega\right)\right),
$$

where $\mathbb{1}$ denotes the indicator function, i.e., for some event $E \subseteq \Omega$ and some state $\omega \in \Omega$ :

$$
\mathbb{1}_{E}(\omega):= \begin{cases}1, & \text { if } \quad \omega \in E, \\ 0, & \text { if } \quad \omega \in \neg E,\end{cases}
$$

where $\neg E$ denotes the complement of $E$. Note that $\mathbb{1}_{E}(\omega)=\pi(E \mid \omega)$.

Assuming that $i$ is risk-averse, she prefers not participating at the states that yield zero expected payoff. Let

$$
R_{i}:=\left\{\omega \in \Omega: i \in S(\omega) \Rightarrow E\left[V_{i} \mid P_{i}(\omega)\right]>0\right\} .
$$

denote the set of states where $i$ rejects participation whenever the expected payoff is not positive. If $i$ is risk-averse, it follows that $R_{i}=\Omega$. Note that the converse is not true as the condition $R_{i}=\Omega$ does not specify $i$ 's risk-attitude in general, e.g., it does not say anything about $i$ 's preferences over two lotteries which yield the same, strictly positive expected payoff. Henceforth, we assume that all agents are risk-averse.

Let $W$ be the set of states where the winner does participate in the bet, i.e.,

$$
W:=\left\{\omega \in \Omega: \sum_{j \in S(\omega)} \mathbb{1}_{G_{j}}(\omega)=1\right\} .
$$

The following intermediate result shows that only states in $W$ are relevant for $i$ 's decision on whether to participate or not. The reason is that states in $\neg W$ yield payoff equal to 0 for everybody, regardless their action: The winner does not participate and therefore every participant receives her fee back after the realization of the state and the announcement of the winner.

Lemma 1 For all $\omega \in R_{i}$, agent $i$ 's expected payoff is equal to

$$
E\left[V_{i} \mid P_{i}(\omega)\right]= \begin{cases}\pi\left(W \mid P_{i}(\omega)\right) E\left[V_{i} \mid P_{i}(\omega) \cap W\right], & \text { if } i \in S(\omega), \\ 0, & \text { if } i \notin S(\omega) .\end{cases}
$$

Proof If $i \notin S(\omega)$ it follows from $\mathcal{P}_{i}$-measurability of $a_{i}$ that $E\left[V_{i} \mid P_{i}(\omega)\right]=0$. If $i \in S(\omega)$, it follows from $\omega \in R_{i}$ that $E\left[V_{i} \mid P_{i}(\omega)\right]>0$. Thus, there is at least one state in $P_{i}(\omega)$ where $i$ wins, which implies that $P_{i}(\omega) \cap W \neq \varnothing$. Now, consider the following two possible cases: (i) $P_{i}(\omega) \cap \neg W=\varnothing$, or (ii) $P_{i}(\omega) \cap \neg W \neq \varnothing$. In the first case, it is straightforward that $P_{i}(\omega) \subseteq W$, which implies

$$
\begin{aligned}
E\left[V_{i} \mid P_{i}(\omega)\right] & =E\left[V_{i} \mid P_{i}(\omega) \cap W\right] \\
& =\pi\left(W \mid P_{i}(\omega)\right) E\left[V_{i} \mid P_{i}(\omega) \cap W\right] .
\end{aligned}
$$

In the second case, it follows from the law of total probability that 


$$
\begin{aligned}
E\left[V_{i} \mid P_{i}(\omega)\right]= & \pi\left(P_{i}(\omega) \cap W \mid P_{i}(\omega)\right) E\left[V_{i} \mid P_{i}(\omega) \cap W\right] \\
& +\pi\left(P_{i}(\omega) \cap \neg W \mid P_{i}(\omega)\right) E\left[V_{i} \mid P_{i}(\omega) \cap \neg W\right] .
\end{aligned}
$$

At every $\omega^{\prime} \in \neg W$ the winner does not participate, and therefore $V_{i}\left(\omega^{\prime}\right)=0$, implying that $E\left[V_{i} \mid P_{i}(\omega) \cap \neg W\right]=0$, which completes the proof.

\subsection{Results}

It is commonly known that $\kappa$ individuals participate in the bet, whenever $s(\omega)=\kappa$ for every $\omega \in M\left(\omega^{*}\right)$. Clearly, common knowledge of how many people participate is weaker than common knowledge of who participates. That is, if $\kappa$ is commonly known, $i$ does not necessarily know who she is playing against. The following example illustrates such a situation.

Example 1 Consider the state space $\Omega=\left\{\omega_{1}, \omega_{2}\right\}$, and the set of participants $N=$ $\{1,2,3\}$. Let 1 and 2 know the true state, i.e., $P_{i}(\omega)=\{\omega\}$ for $i=1,2$ and for all $\omega \in \Omega$, whereas 3 has no private information, i.e., $P_{3}(\omega)=\Omega$ for all $\omega \in \Omega$. Suppose that 1 and 3 participate at $\omega_{1}$, whereas 2 and 3 participate at $\omega_{2}$. Clearly, it is common knowledge that there are two participants, as exactly two people are willing to bet at every $\omega \in \Omega$. However, the identity of the participants is not commonly known: 3 does not know who she is playing against, as she cannot distinguish between the two states; she only knows that she is betting against exactly one agent, but she does not know who.

When the identity of the participants is commonly known, by definition it is the case that for some $I \subseteq N$ we obtain $S(\omega)=I$ for all $\omega \in M\left(\omega^{*}\right)$, and therefore those who reject participation at $\omega^{*}$, also do so at all $\omega \in M\left(\omega^{*}\right)$. Then, no bet can take place.

Proposition 1 If all agents are risk-averse, and the set of participants is commonly known, then nobody participates. If $R_{i}=\Omega$ for all $i \in N$, and $S(\omega)=I$ for every $\omega \in M\left(\omega^{*}\right)$, then $I=\varnothing$.

Proof Suppose that $I \neq \varnothing$. It follows from risk-aversion that $E\left[V_{i} \mid P_{i}(\omega)\right]>0$ for every $i \in I$ and for all $\omega \in M\left(\omega^{*}\right)$. Since $M\left(\omega^{*}\right)$ is $\mathcal{P}_{i}$-measurable, summing over the information cells $P_{i}(\omega) \subseteq M\left(\omega^{*}\right)$ implies-by the law of total probabilitythat $E\left[V_{i} \mid M\left(\omega^{*}\right)\right]>0$ for all $i \in I$. Now, sum again over all $i \in I$, and obtain $E\left[\sum_{i \in I} V_{i} \mid M\left(\omega^{*}\right)\right]>0$, which contradicts the following statement: Let $\kappa$ be the cardinality of $I$. For an arbitrary $\omega \in M\left(\omega^{*}\right)$,

$$
\begin{aligned}
\sum_{i \in I} V_{i}(\omega) & =\sum_{i \in I}\left(\kappa \pi\left(G_{i} \mid \omega\right)-\sum_{j \in I} \pi\left(G_{j} \mid \omega\right)\right) \\
& =\kappa \sum_{i \in I} \pi\left(G_{i} \mid \omega\right)-\kappa \sum_{j \in I} \pi\left(G_{j} \mid \omega\right)=0
\end{aligned}
$$

implying that $E\left[\sum_{i \in I} V_{i} \mid M\left(\omega^{*}\right)\right]=0$, which completes the proof. 
The previous result is not very surprising. Essentially, it is an extension of the no-bet theorem (Sebenius and Geanakoplos 1983) to the case of more than two individuals. What is not trivial, is proving the no-bet theorem in cases where $s\left(\omega^{*}\right)$-rather than $S\left(\omega^{*}\right)$-is common knowledge. As the following result states this is indeed the case, i.e., common knowledge of the number of participants precludes betting.

Theorem 1 If all agents are risk-averse, and the number of participants is commonly known, then nobody participates: If $R_{i}=\Omega$ for all $i \in N$, and $s(\omega)=\kappa$ for every $\omega \in M\left(\omega^{*}\right)$, then $\kappa=0$.

Proof It follows directly from Lemma 1 that if $i \in S(\omega)$ then $i$ 's expected payoff can be rewritten as:

$$
\begin{aligned}
E\left[V_{i} \mid P_{i}(\omega)\right]= & \pi\left(W \mid P_{i}(\omega)\right) \sum_{\omega^{\prime} \in P_{i}(\omega) \cap W} \pi\left(\omega^{\prime} \mid P_{i}(\omega) \cap W\right) \\
& \times\left(s\left(\omega^{\prime}\right) \pi\left(G_{i} \mid \omega^{\prime}\right)-\sum_{j \in S\left(\omega^{\prime}\right)} \pi\left(G_{j} \mid \omega^{\prime}\right)\right) \\
= & \pi\left(W \mid P_{i}(\omega)\right) \sum_{\omega^{\prime} \in P_{i}(\omega) \cap W} \pi\left(\omega^{\prime} \mid P_{i}(\omega) \cap W\right)\left(s\left(\omega^{\prime}\right) \pi\left(G_{i} \mid \omega^{\prime}\right)-1\right),
\end{aligned}
$$

since by definition $\sum_{j \in S\left(\omega^{\prime}\right)} \pi\left(G_{j} \mid \omega^{\prime}\right)=1$ holds whenever $\omega^{\prime} \in W$.

Suppose now that $s(\omega)=\kappa$ for all $\omega \in M\left(\omega^{*}\right)$, where $0<\kappa \leq n$. It follows from Eq. 7 and risk-aversion that

$\sum_{\omega^{\prime} \in P_{i}(\omega) \cap W} \pi\left(\omega^{\prime} \mid P_{i}(\omega) \cap W\right)\left(s\left(\omega^{\prime}\right) \pi\left(G_{i} \mid \omega^{\prime}\right)-1\right)=\kappa \pi\left(G_{i} \mid P_{i}(\omega) \cap W\right)-1>0$

for every $i$ such that $B_{i} \cap M\left(\omega^{*}\right) \neq \varnothing$, and for all $\omega \in B_{i} \cap M\left(\omega^{*}\right)$. Thus, for every such individual—using the fact that $B_{i}$ and $M\left(\omega^{*}\right)$ are $\mathcal{P}_{i}$-measurable-we have

$$
\kappa \pi\left(G_{i} \mid B_{i} \cap M\left(\omega^{*}\right) \cap W\right)>1,
$$

which is obviously equivalent to

$$
\kappa \pi\left(G_{i} \cap B_{i} \mid B_{i} \cap M\left(\omega^{*}\right) \cap W\right)>1 .
$$

Hence, multiplying by the positive $\pi\left(B_{i} \mid M\left(\omega^{*}\right) \cap W\right)$, which is equal to $\pi\left(B_{i} \cap\right.$ $\left.M\left(\omega^{*}\right) \cap W \mid M\left(\omega^{*}\right) \cap W\right)$, it follows that

$$
\kappa \pi\left(G_{i} \cap B_{i} \mid M\left(\omega^{*}\right) \cap W\right)>\pi\left(B_{i} \mid M\left(\omega^{*}\right) \cap W\right) .
$$


Finally, since $\pi\left(B_{i} \mid M\left(\omega^{*}\right) \cap W\right)=0$ for every $i \in N$ such that $B_{i} \cap M\left(\omega^{*}\right)=\varnothing$, summing over all individuals in $N$ yields

$$
\kappa=\kappa \sum_{i \in N} \pi\left(G_{i} \cap B_{i} \mid M\left(\omega^{*}\right) \cap W\right)>\sum_{i \in N} \pi\left(B_{i} \mid M\left(\omega^{*}\right) \cap W\right)=\kappa,
$$

which completes the proof by contradiction.

Note that all our results are directly extended to gambles that do not cover $\Omega$, i.e., we may allow for the possibility of a subset $G_{0}$ of $\Omega$ where no participant wins, and all participants receive their entry fee back. These states are payoff irrelevant, since they can be treated as a subset of $\neg W$.

It is straightforward to extend our main result to negative sum bets, where the payoff depends on the number of participants, but not on who participates. Agent $i$ 's payoff function at $\omega$ becomes equal to the following expression in this case:

$$
\begin{aligned}
\hat{V}_{i}(\omega) & =\mathbb{1}_{B_{i}}(\omega)\left(s(\omega) \mathbb{1}_{G_{i}}(\omega)-1\right) \\
& =\mathbb{1}_{B_{i}}(\omega)\left(s(\omega) \pi\left(G_{i} \mid \omega\right)-1\right) .
\end{aligned}
$$

If $i$ participates, she has to pay the 1 unit entrance fee, even if the winner decides to stay out. Clearly, $\hat{V}_{i}(\omega) \leq V_{i}(\omega)$ for all $\omega \in \Omega$, and therefore $i$ participates less often than she would do in a zero-sum bet, as the one analyzed above, and therefore common knowledge of $s\left(\omega^{*}\right)$ again precludes the bet. The proof is similar to the one of Theorem 1.

Acknowledgements I am indebted to Alpaslan Akay, Geir Asheim, Martin Dufwenberg, Amanda Friedenberg, John Geanakoplos, Andrés Perea, Jeff Steif, Mark Voorneveld, Jörgen Weibull, an anonymous referee and an associate editor for valuable comments and fruitful discussions. I would also like to thank the University of California, Berkeley and the Stockholm School of Economics for their hospitality while working on this project. The financial support from the Adlerbertska Research Foundation is gratefully acknowledged.

Open Access This article is distributed under the terms of the Creative Commons Attribution Noncommercial License which permits any noncommercial use, distribution, and reproduction in any medium, provided the original author(s) and source are credited.

\section{References}

Aumann RJ (1976) Agreeing to disagree. Ann Stat 4:1236-1239

Geanakoplos J, Polemarchakis H (1982) We can't disagree forever. J Econ Theory 28:192-200

Hanson R (1998) Consensus by identifying extremists. Theory Decis 44:293-301

Hanson R (2002) Disagreement is unpredictable. Econ Lett 77:365-369

Milgrom P, Stokey N (1980) Information, trade, and common knowledge. J Econ Theory 26:17-27

Nielsen LT (1995) Common knowledge of a multivariate aggregate statistic. Int Econ Rev 36:207-216

Nielsen LT, Brandenburger A, Geanakoplos J, McKelvey R, Page T (1990) Common knowledge of an aggregate of expectations. Econometrica 58:1235-1239

Sebenius J, Geanakoplos J (1983) Don't bet on it: contingent agreements with asymmetric information. J Am Stat Assoc 78:424-426 